\title{
Coordinated Electron Energy Loss and Energy Dispersive X-ray Spectroscopies of Organic Matter from Asteroids
}

\author{
Rhonda Stroud $^{1}$, Bradley De Gregorio ${ }^{1}$ and Conel Alexander ${ }^{2}$ \\ ${ }^{1}$ U.S. Naval Research Laboratory, Washington, District of Columbia, United States, ${ }^{2}$ Carnegie Institution of \\ Washington, United States
}

The study of organic matter from asteroids enables planetary scientists to identify remnant organic building blocks of our solar system, and investigate how those materials have evolved over time due to heating, and interactions with aqueous fluids and minerals [1]. The organic matter is available for laboratory study as a minor component (0-4 wt.\%) of the matrices of primitive meteorites. Each meteorite is classified according to the level of thermal and aqueous processing manifest in the bulk mineralogy, but their specific parent asteroids are generally not known. Samples returned by spacecraft from two carbonaceous near-Earth asteroids, Bennu and Ryugu, also will soon be available for study.

Meteoritic acid-insoluble organic matter (IOM) is a macromolecular polymer distributed throughout the meteorite matrices as discrete micron-to-sub-micron particles, as clusters of particles, as coatings on inorganic particles, and as vein deposits extending several microns. In order to perform analysis representative of its bulk properties, the IOM is isolated from a particular host meteorite through a series of chemical dissolution steps [1]. The resultant powder can be prepared for transmission electron microscopy, or other microanalyses, by ultramicrotomy. Alternatively, focused ion beam lift-out sections of the meteorite matrix can be extracted, in order to examine site-specific regions of the IOM in the petrographic context of the meteorite matrix. Scanning transmission electron microscope (STEM) imaging and spectroscopy are attractive methods for characterization of the nanoscale variation of the IOM in elemental composition and $\mathrm{C}$ functional chemistry, although electron beam-induced alteration is a significant concern $[2,3]$.

In this study, we use STEM imaging, electron energy loss spectroscopy (EELS) and energy dispersive x-ray spectroscopy (EDS) to characterize IOM from meteorites with distinct alteration histories, including Allan Hill 77307 (CO3), Elephant Morraine 92042 (CR2), and Murchison (CM2). Details of the sample processing are described elsewhere [1]. Sections for TEM analysis were prepared by $\mathrm{S}$ embedding and microtome sectioning to thicknesses of $30 \mathrm{~nm}-70 \mathrm{~nm}$, and then placed over holes in non-continuous C support films. Data were collected with the Naval Research Lab (NRL) Nion UltraSTEM 200-X operated at $60 \mathrm{kV}$ and probe currents ranging from 25 to $100 \mathrm{pA}$, with a nominal probe size of $1.5 \AA$. The NRL UltraSTEM is equipped with a Gatan Enfinium ER energy loss spectrometer, and a Bruker windowless, 0.7 sr SDD EDS system. Simultaneous EELS-EDS spectrum images (SIs) were acquired in Digital Micrograph in a single pass at a spatial resolution of $\sim 25 \mathrm{~nm} / \mathrm{px}$ and $0.02 \mathrm{eV} / \mathrm{ch}$ energy dispersion, with dwell times up to $8 \mathrm{~s} / \mathrm{px}$, in order to observe details of the $\mathrm{C}-\mathrm{K}$ edge fine structure, and correlate it with elemental composition variations. Sub-pixel scanning at 16x was selected to spread the beam dose and reduce local damage. Higher spatial resolution EDS SIs $(\sim 2 \mathrm{~nm} / \mathrm{px})$ were obtained after the coordinated SI acquisitions to look for more fine-scale compositional variation. For assessment of the alteration of the $\mathrm{C}$ functional chemistry for the IOM with different alteration histories, a subset of the samples were first examined using x-ray absorption near edges spectroscopy (XANES) of the C-K edge, at the 5.3.2.2 polymer STXM beamline at the Advanced Light Source, prior to STEM analysis (Fig. 1). Our results show a qualitative match of the EELS and XANES data, and correlation of the N and O EDS signal in regions with corresponding features in the C-K fine structure (Fig. 2) . However, comparison of the EELS and XANES data shows that even under "gentle" measurement conditions, loss or alteration of $\mathrm{H}$ bearing aliphatic functional groups occurs, even for samples with a history of thermal alteration. Use of direct electron detector technology for EELS acquisition in order to obtain better quality spectra at lower beam doses 
could significantly reduce the damage and improve the quantitative EELS-XANES match. For the most sensitive samples, use of a cold stage to prevent $\mathrm{H}$ loss is likely beneficial [4].
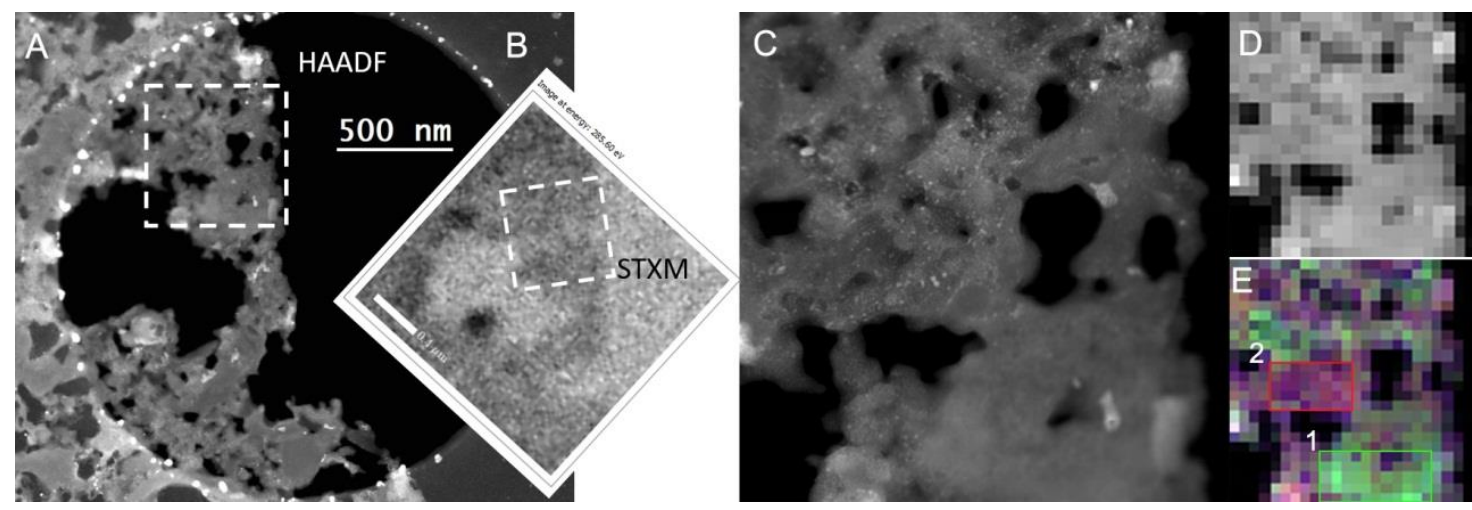

Figure 1. (A) STEM-HAADF of insoluble organic matter from EET 92042 (CR2) meteorite. (B) STXM image at $285.6 \mathrm{eV}$. (C) HAADF Analog image for EELS-EDS spectrum image. (D) $285 \mathrm{eV}$ EELS SI slice. (E) RBG overlay of C, N, and O EDS windows from EDS SI. The EELS and EDS spectra from ROIs 1 and 2 are shown in Fig. 2.
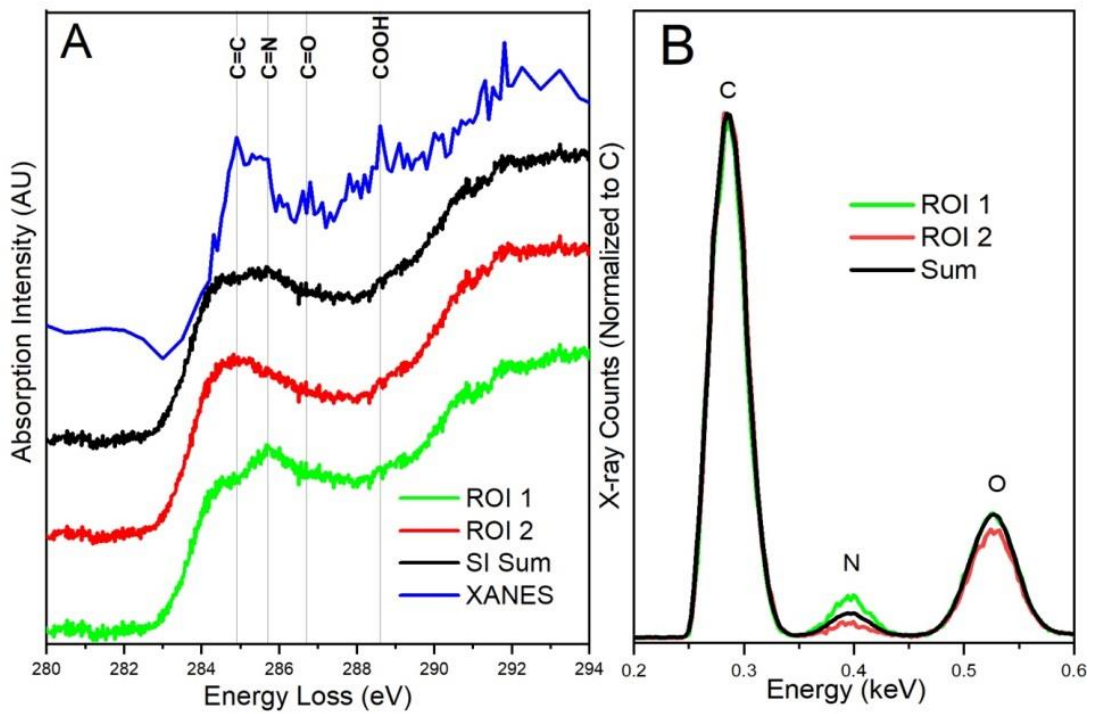

Figure 2. (A) Comparison of C-K edge EELS and XANES from areas defined in Fig. 1. (B) Corresponding EDS data.

\section{References}

[1] C. M. O’D. Alexander et al. Chemie der Erde 77 (2007), p. 227.

[2] C. Le Guillou et al. Geochemica et Cosmochemica Acta 131 (2014), p. 368.

[3] C. Vollmer et al. Scientific Reports 10 (2020), p. 20251.

[4] This work is funding by NASA awards 80HQRT19T0038 and 80NSSC19M0215. The ALS beamline is supported by the U.S. DOE Office of Science (contract DE-AC02-05CH11231). 